\title{
Validation and Reference Ranges of Hair Cortisol Measurement in Healthy Children
}

\author{
Gerard Noppe ${ }^{a, b, d} \quad$ Elisabeth F.C. Van Rossum ${ }^{a, d}$ Jan Willem Koper ${ }^{a}$ \\ Laura Manenschijn $^{a}$ G. Jan Bruining ${ }^{b}$ Yolanda B. de Rijke ${ }^{a, c}$ \\ Erica L.T. van den Akkerb, d \\ a Section of Endocrinology, Department of Internal Medicine, ${ }^{b}$ Department of Pediatrics, Sophia Children's Hospital, \\ and ${ }^{\mathrm{C}}$ Department of Clinical Chemistry, Erasmus MC, University Medical Center, and ${ }^{\mathrm{d}}$ Obesity Center CGG, Erasmus \\ MC and Sint Franciscus Gasthuis, Rotterdam, The Netherlands
}

\section{Key Words}

Hair cortisol · Reference ranges · Children · Age · Gender .

Puberty

\begin{abstract}
Background: Cortisol is produced in a circadian rhythm controlled by the hypothalamus-pituitary-adrenal axis, making it cumbersome to measure long-term cortisol exposure. Hair has proven to be a reliable matrix for long-term cortisol measurement in adults and can be used as diagnostic tool for (cyclic) Cushing's syndrome. The diagnostic applicability in children has not been studied, nor have the effects of development and hair care been evaluated in children. We aimed to establish reference ranges of hair cortisol concentrations (HCC) in healthy children and to evaluate the effects of age, gender, puberty and characteristics of hair care. Methods: In 128 healthy children aged 4-14 years, HCC were measured in a small 3-cm hair lock from the back of the head. Results: HCC increased with age $(p=0.04)$ up to age 10 years, with a mean of 5.0, 5.8, 6.8 and $8.5 \mathrm{pg} / \mathrm{mg}$ at age $4-5,6-7,8-9$ and 10-14 years, respectively. Children aged 4-7 years had significantly lower HCC compared to healthy adults $(p=0.007)$. We did not find any influence of gender, puberty or hair care characteristics on hair cortisol. Conclusion: HCC can be reli-
\end{abstract}

ably measured in childhood, and reference ranges increase with age. HCC in children are not dependent on hair care or hair characteristics.

(c) 2014 S. Karger AG, Basel

\section{Introduction}

The glucocorticoid stress hormone cortisol is the end product of the hypothalamus-pituitary-adrenal (HPA) axis. Both a deficiency and an excess of cortisol have detrimental effects, resulting in both physical and psychiatric symptoms as manifested in Cushing's syndrome and Addison's disease. Cortisol is measurable in several matrices, each with its own merits and drawbacks. Cortisol measurements in serum and saliva provide a snapshot indication of cortisol concentrations but are subject to the circadian rhythm, pulsatile excretion and acute stress, thereby impeding interpretation [1]. Urinary free cortisol is less subject to these drawbacks and provides a cortisol concentration spanning $24 \mathrm{~h}$. However, 24-hour urine collection is often cumbersome and unreliable. Moreover, most of the deleterious effects of hyper- and hypocortisolism are mediated by long-term exposure. A more suitable matrix for long-term cortisol measurement is

Erica L.T. van den Akker, MD, PhD 
scalp hair. Hair is readily accessible, sampling is non-invasive, samples can easily be stored at room temperature and cortisol can be measured using a relatively simple method. Measurement of hair cortisol has been shown to be a valuable diagnostic tool in situations of extreme hypercortisolism such as seen in Cushing's syndrome [2], but also hair cortisol concentrations (HCC) within the physiological range have been associated with the metabolic syndrome [3], obesity [4] and cardiovascular disease [5]. Recently, HCC are being explored as a stress biomarker in children [6-8].

Hair cortisol measurements have been validated in adults $[2,9]$ and so far most studies measuring hair cortisol were in adults. A prerequisite to study hair cortisol in children is the availability of reference values, according to age, gender and pubertal stages. These have hitherto not been published.

In this study we aimed at measuring HCC in healthy children aged 4-14 years to establish reference ranges. Furthermore, we evaluated factors potentially influencing HCC such as age, gender, puberty, use of hair products, washing frequency and distance to scalp variation.

\section{Methods}

Healthy children were enrolled during visitation of primary schools and from the siblings of children attending the pediatric outpatient clinic. Children were excluded if they were using glucocorticoids, both systemic and topical, or medication known to alter glucocorticoid metabolism. In total, HCC were measured in 128 children aged 4 through 14 years. Only 2 children were non-Caucasoid by appearance. Parents were requested to fill out a questionnaire about general health, the use of medication and the hair care characteristics of their child. Hair care characteristics which were queried are hair washing frequency $(<1,1-2,3-4$ or $>4$ times/ week), the use of hair products on the day of sampling (yes/no and type: none, mousse, gel, wax, spray, other), frequent sweating at the scalp (yes/no) and hair treatment in the last 6 months (bleaching, dyeing, perming). Hair color was noted during preparation for analysis. In all children hair samples were obtained and anthropometrics were measured by one trained staff member as follows: standing height was measured in centimeters with the precision of $1 \mathrm{~mm}$ with a wall-mounted stadiometer. Body weight was measured in kilograms with the precision of $100 \mathrm{~g}$ with electric scales. Waist circumference (WC) and hip circumference (HC) were assessed in centimeters with the precision of $1 \mathrm{~mm}$, taken on bare skin with both feet standing on the floor. The WC was measured midway between the lowest rib and the upper side of the pelvis. The HC was measured at the height of the widest range of the buttocks and the upper side of the os pubis. WC and HC measurements were taken twice and the mean was used as outcome. In children aged 7 and above Tanner puberty staging was assessed by a trained research nurse. Puberty was defined as Tanner stage $M$ $>1$ in girls and testis volume $\geq 4 \mathrm{ml}$ in boys. Approval was given by the local medical ethics committee, written informed consent was obtained from parents and informed assent from participants.

\section{Hair Collection}

Of each participant, a lock of hair was cut from the posterior vertex as close to the scalp as possible using small surgical scissors. This area has been shown to have the lowest interlock variation [10]. Hair locks were taped to a paper form with scalp end marking and stored at room temperature until analysis.

\section{Hair Analysis}

Hair samples were prepared and analyzed as described previously [9]. Briefly, the proximal 3-cm hair segment was weighed (samples approx. between 25 and $40 \mathrm{mg}$ ) and minced using small surgical scissors. The hair was extracted in $1 \mathrm{ml}$ methanol for $16 \mathrm{~h}$ at $52^{\circ} \mathrm{C}$ with gentle shaking. The extract was transferred to a glass tube and the methanol was evaporated at $37^{\circ} \mathrm{C}$ under a constant stream of nitrogen and reconstituted in $250 \mu \mathrm{l}$ phosphate-buffered saline $\mathrm{pH}$ 8.0. After vortexing, the samples were analyzed using a commercially available cortisol ELISA kit (DRG Instruments, $\mathrm{GmbH}$, Marburg, Germany) following the manufacturer's directions. The cross-reactivity as reported by the manufacturer is as follows: corticosterone $29.0 \%$, cortisone $3.0 \%$, 11-deoxycortisol $<1.0 \%, 17-\mathrm{OH}$ progesterone $<0.5 \%$, testosterone $<0.10 \%$, and estradiol $<0.10 \%$.

\section{Assay Variation}

The intra- and interassay variations were analyzed on internal quality controls used for routine saliva cortisol measurement, measured in duplicate on five consecutive assays. The intra-assay coefficients of variance $(\mathrm{CV})$ were $3.1 \%$ at $4.4 \mathrm{ng} / \mathrm{ml}, 2.3 \%$ at 21.3 , and $2.6 \%$ at $35.0 \mathrm{ng} / \mathrm{ml}$. The interassay CVs were $7.0,2.3$, and $8.2 \%$, respectively.

\section{Variation within Lock between Proximal and Distal Segments}

(Distance to Scalp Variation)

To asses intra-individual variation over time, we measured HCC in 15 participants in the distal $3-\mathrm{cm}$ segment adjacent to the proximal $3 \mathrm{~cm}$ (from 3 to $6 \mathrm{~cm}$ distal from the scalp) of hair within a single hair lock. These participants were randomly selected with the requirement that hair length was at least $6 \mathrm{~cm}$. The Spearman correlation between the two measurements is reported.

\section{Statistical Analysis}

Statistical analyses were performed using SPSS 20.0 (IBM Corp., Armonk, N.Y., USA). HCC were $\log _{10}$-transformed to achieve normality. Anthropometrics standard deviation (SD) scores were calculated based on the 1997 Dutch nationwide growth study [11] using Growth Analyser RCT (Growth Analyser BV, Rotterdam, The Netherlands). WC to height ratio was calculated by dividing WC $(\mathrm{cm})$ by height $(\mathrm{cm})$. Relations between hair cortisol and age and anthropometrics were analyzed by linear regression. Age-specific reference ranges are based on linear regression 95\% prediction intervals. Differences in HCC between age groups were tested using Student's t test. Proximal and distal hair samples were compared using Spearman's correlation and differences were analyzed using Wilcoxon signed-rank test. Effects of hair care and hair characteristics on cortisol concentrations were assessed using one-way analysis of variance (ANOVA).
Noppe/Van Rossum/Koper/Manenschijn/ Bruining/Rijke/van den Akker 


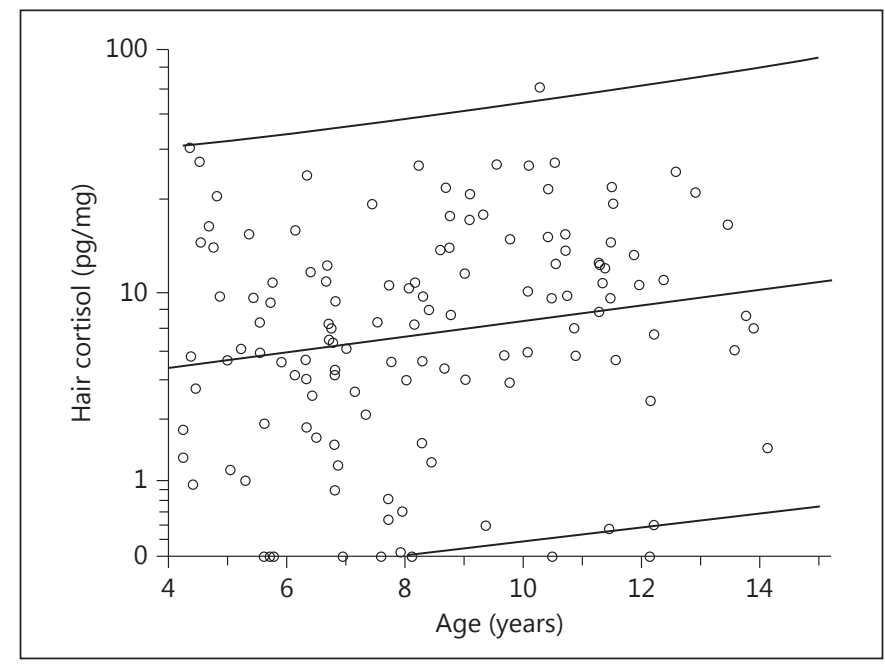

Fig. 1. Hair cortisol distribution over age. Each dot represents a single individual. Lines show linear regression and limits of 95\% prediction intervals.

Table 1. General characteristics of study participants $(n=128)$

\begin{tabular}{lc}
\hline Mean age (range), years & $8.4(4.25-14.13)$ \\
Female, $\mathrm{n}(\%)$ & $65(50.8)$ \\
Mean BMI SD score (SD) & $-0.25(0.99)$ \\
Mean WC SD score (SD) & $0.72(0.83)$ \\
Puberty, n (\%) & $26(20.3)$ \\
\hline
\end{tabular}

\section{Results}

General characteristics of participants are shown in table 1. HCC increased significantly with age (log-transformed $\beta=0.031, r=0.18, p=0.04$; fig. 1 ), which is emphasized by the significantly lower HCC in children aged 4-7 years compared to 141 healthy adults as previously measured [9] (mean and 95\% CI $4.6(3.3,6.1)$ vs. 7.5 (6.1, 9.1) $\mathrm{pg} / \mathrm{mg}, \mathrm{p}=0.008)$ and compared to children aged $8-14$ years (mean and $95 \%$ CI $4.6(3.3,6.2)$ vs. 9.0 (6.9, 11.6) $\mathrm{pg} / \mathrm{mg}, \mathrm{p}=0.001)$. Cortisol concentrations were not different between children aged 8-14 and adults $(\mathrm{p}=$ $0.14)$. Neither puberty nor gender influenced HCC ( $\mathrm{p}=$ 0.88 and $\mathrm{p}=0.78$, respectively). WC showed a significant association with HCC (log-transformed $\beta=0.13, \mathrm{r}=0.19$, $\mathrm{p}=0.04$, adjusted for age; fig. 2); the association between waist to height ratio did not reach statistical significance

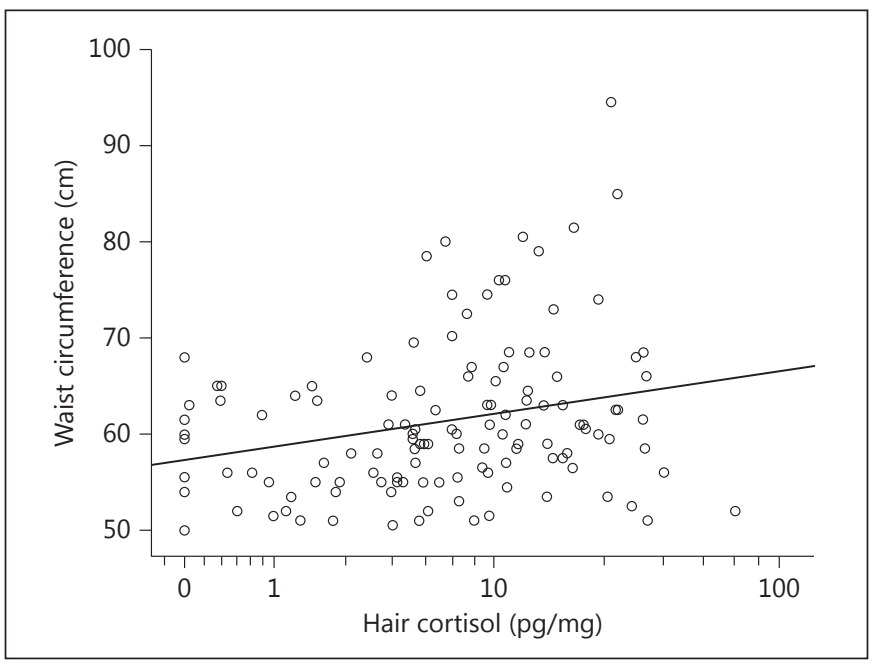

Fig. 2. WC plotted against HCC. The line shows unadjusted correlation. Linear regression adjusted for age: log-transformed $\beta=$ $0.13, \mathrm{p}=0.04$.

Table 2. HCC (pg/mg) reference ranges per age category

$\begin{array}{ll}\text { Age } 4-5(\mathrm{n}=27) & 5.0(0-43.4) \\ \text { Age } 6-7(\mathrm{n}=34) & 5.8(0-49.1) \\ \text { Age } 8-9(\mathrm{n}=27) & 6.7(0.1-56.0) \\ \text { Age } 10-14(\mathrm{n}=40) & 8.5(0.3-69.5)\end{array}$

Reference ranges are described by linear regression mean and 95\% prediction interval limits per age category.

(log-transformed $\beta=0.16, r=0.19, p=0.07$, adjusted for age). Hair cortisol was not correlated with BMI ( $p=0.50$, adjusted for age).

\section{Age-Specific Reference Ranges}

Age-specific hair cortisol reference ranges based on linear regression 95\% prediction intervals are listed in table 2 . HCC in children above age 10 were not significantly different from adults and were hence pooled in one category.

\section{Hair Care}

Hair care characteristics in terms of washing frequen$c y$, hair product use or type of hair product did not influence hair cortisol content $(\mathrm{p}=0.33, \mathrm{p}=0.73$, and $\mathrm{p}=0.22$, respectively), nor was hair cortisol content affected by hair color or frequent sweating at the scalp ( $p=0.72$ and $\mathrm{p}=0.25$, respectively). Adjustment for age did not change 
these results. None of the children participating in the current study dyed, bleached or permed their hair.

\section{Distance to Scalp Variation}

Intra-individual differences in hair cortisol measurements between the proximal $3-\mathrm{cm}$ hair segment and a distal 3-cm hair segment (3-6 cm distal from scalp) measured in 15 children (male $\mathrm{n}=8$ ) showed a significant correlation (Spearman $r=0.76, p=0.001$ ). No significant difference in HCC between proximal and distal segments was observed $(\mathrm{p}=0.43)$.

\section{Discussion}

HCC in healthy children were readily measurable, and increased significantly with age. In other matrices, e.g. serum or saliva, the relation between age and cortisol concentrations has yielded conflicting results. Knutsson et al. [12] found no effects of age on serum cortisol in a study in 235 healthy children. However, two larger studies on serum cortisol in 762 and 1,482 healthy children aged $0-18$, respectively, reported high cortisol variance in neonates and infants, after which cortisol rapidly decreased to subsequently increase steadily with age up to adulthood [13, 14]. More specifically, the median serum cortisol concentration in the study by Elmlinger et al. [14] was $218 \mathrm{nmol} / \mathrm{l}$ in girls and $253 \mathrm{nmol} / \mathrm{l}$ in boys aged $4-6$ years and rose steadily up to $466 \mathrm{nmol} / \mathrm{l}$ (girls) and $440 \mathrm{nmol} / \mathrm{l}$ (boys) in 14-year-olds. These study results support our findings in hair cortisol. Very few studies report hair cortisol measurement in children. Dettenborn et al. [15] reported a decrease in hair cortisol over age in 28 children 1-9 years of age. This seems contradictory to our findings, however the numbers per age category in that study are low and the sample mainly composed of children under 4 years of age (mean age 3.6 years) including only 8 children aged above 5 , whereas we studied children 4-14 years old (mean age 8.4 years). The differences may be explained in the relative overrepresentation of very young children, as serum cortisol show high and variable concentrations during infancy [13]. The high variability in HCC at young age has also been demonstrated by Karlén et al. [16], measuring hair cortisol in children of $1,3,5$, and 8 years of age. HCC showed a large variation in the very young, which decreased with increasing age. However, no clear difference was visible between the age of 5 and 8 years. HCC may be further elevated during the first years of life when hair morphology and growth is subject to major changes [17]. After the age of 2 , hair has been observed to increase in cross-sectional surface area and in the percentage of medullated hair with age up to puberty [18]. The influence of these morphological changes on hair cortisol content is unknown. Theoretically, linear hair growth rate may also influence HCC. Research on hair growth rate in healthy children is scarce. Growth rates of $0.3-0.4 \mathrm{~mm} /$ day have been reported [17]. One study suggests a slightly higher hair growth rate in childhood in boys aged 3-9 years [17, 19]. It seems unlikely that the hair growth rate contributes significantly to hair cortisol content; as hair grows slowly there is ample time for circulating cortisol to achieve an equilibrium at the hair follicle and to completely diffuse into the forming hair shaft, even in the case of a slight increase in growth rate. However, differences in growth rate may have consequences if consecutive segments of hair are used to construct a timeline of cortisol exposure. For such applications, it would be useful to have more data about the hair growth rate in children.

To validate hair cortisol measurement in children, factors that could influence cortisol concentrations were studied. HCC were not affected by hair color, hair washing frequency or use of hair products. These findings are in line with previously described findings in adults [9]. The effect of topical use of glucocorticoid treatment, e.g. hydrocortisone cream, was not assessed, as this was a criterion for exclusion. Despite recent evidence showed extensive sweating at the scalp may increase HCC [20], no effect of frequent sweating at the scalp as reported through a questionnaire was found in the present study. However, noting extensive sweating at the time of sampling as suggested by the authors was not performed. Hair segments up to $6 \mathrm{~cm}$ from the scalp were measured in the current study. No significant difference in HCC was found between the proximal $(0-3 \mathrm{~cm})$ and distal $(4-6 \mathrm{~cm})$ hair segments, and distal and proximal hair segments showed a high correlation, supporting the previous evidence of stable incorporation of cortisol in hair. With the current method, HCC can be reliably measured at least up to 6 $\mathrm{cm}$. As none of the children participating in the current study had undergone any hair treatment, e.g. dyeing, straightening or perming, the effects of these treatments could not be assessed. The same applies to ethnicity, as almost all participants were Caucasian.

Stressing the value of hair cortisol as a reflection of long-term systemic cortisol exposure, hair cortisol was significantly correlated with WC in children, since visceral fat redistribution is one of the hallmarks of cortisol tissue effects. Also in adults, positive correlations between waist and hair cortisol levels have been demonstrated [3, 
9]. In addition, strong evidence was recently presented that HCC in adults are related to metabolic syndrome and cardiovascular disease $[3,5]$.

Several limitations of the current study need to be mentioned. First, the population is primarily Caucasian, limiting the applicability of the reference ranges to other ethnicities. However, hair color has been observed not to influence HCC [15], so there are no clear indications that HCC is different in other ethnicities. Additionally, the current reference ranges do not cover all pediatric age categories. The findings described here and in other studies [13] underline the need for reference ranges measured in all ages, rather than extrapolating results to other ages. Hair cortisol measurement in children under the age of 4 years deserves extra attention, as hair characteristics in this age group change dramatically [17-19]. Although figure 1 suggests HCC increases up to approximately the age of 10 , no distinct cut-off age at which the increase in HCC levelled off could be determined in the current study using a linear regression model. HCC measurement using different methods for extraction or different ELISA kits render different results $[10,15]$, and while immunoassays will remain in use for HCC measurement, especially in settings where equipment and money are scarce, methods with a higher sensitivity such as gas and liquid chromatography mass spectrometry will eventually be widely employed. The comparison of results between varying methods is an important focus for future research [21].

A strength of this study is the sample size, exceeding 20 children per 2-year age group with an even distribu- tion in gender. The additional validation of these data and this technique is supported by the information on pubertal staging and on potential confounders. With these reference data we have a foundation for further research on the value of using HCC in pediatric clinical practices.

\section{Conclusion}

HCC in childhood can be reliably measured. HCC reference ranges increase with age between 4 and 9 years of age until concentrations comparable to adults are reached in puberty. This study emphasizes the need for age-specific reference ranges. Hair cortisol measurement has the potential of becoming a valuable tool in diagnosing HPA diseases in children in the future. Further research is needed to clarify its value in pediatric clinical practice and research.

\section{Acknowledgements}

The authors are grateful to Ms. Margriet E. Bisschoff, research nurse, Erasmus MC, Rotterdam, The Netherlands, for her help in participant recruitment and measurements. We also thank the children and their parents for their participation. This research has been funded by NIH Thrasher Fund grant TRF11643.

\section{Disclosure Statement}

The authors have no conflicts of interest to disclose.

\section{References}

1 Carroll TB, Aron DC, Findling JW, Tyrrell B: Glucocorticoids and adrenal androgens; in Gardner DG, Shoback D (eds): Greenspan's Basic and Clinical Endocrinology. New York, McGraw-Hill, 2011, vol 9.

2 Manenschijn L, Koper JW, van den Akker EL, de Heide LJ, Geerdink EA, de Jong FH, Feelders RA, van Rossum EF: A novel tool in the diagnosis and follow-up of (cyclic) Cushing's syndrome: measurement of long-term cortisol in scalp hair. J Clin Endocrinol Metab 2012;97:E1836-E1843.

-3 Stalder T, Kirschbaum C, Alexander N, Bornstein SR, Gao W, Miller R, Stark S, Bosch JA, Fischer JE: Cortisol in hair and the metabolic syndrome. J Clin Endocrinol Metab 2013;98: 2573-2580.

Hair Cortisol Validation in Children
4 Veldhorst MA, Noppe G, Jongejan MH, Kok CB, Mekic S, Koper JW, van Rossum EF, van den Akker EL: Increased scalp hair cortisol concentrations in obese children. J Clin Endocrinol Metab 2014;99:285-290.

5 Manenschijn L, Schaap L, van Schoor NM, van der Pas S, Peeters GM, Lips P, Koper JW, van Rossum EF: High long-term cortisol levels, measured in scalp hair, are associated with a history of cardiovascular disease. J Clin Endocrinol Metab 2013;98:2078-2083.

6 Groeneveld MG, Vermeer HJ, Linting M, Noppe G, van Rossum EF, van IJzendoorn MH: Children's hair cortisol as a biomarker of stress at school entry. Stress 2013;16:711-715.

7 Hostinar CE, Gunnar MR: Future directions in the study of social relationships as regulators of the HPA axis across development. J Clin Child Adolesc Psychol 2013;42:564-575.
8 Vanaelst B, Huybrechts I, Bammann K, Michels N, de Vriendt T, Vyncke K, Sioen I, Iacoviello L, Gunther K, Molnar D, Lissner L, Rivet N, Raul JS, de Henauw S: Intercorrelations between serum, salivary, and hair cortisol and child-reported estimates of stress in elementary school girls. Psychophysiology 2012;49:1072-1081.

-9 Manenschijn L, Koper JW, Lamberts SW, van Rossum EF: Evaluation of a method to measure long-term cortisol levels. Steroids 2011; 76:1032-1036.

10 Sauve B, Koren G, Walsh G, Tokmakejian S, Van Uum SH: Measurement of cortisol in human hair as a biomarker of systemic exposure. Clin Invest Med 2007;30:E183-E191. 
11 Fredriks AM, van Buuren S, Burgmeijer RJ, Meulmeester JF, Beuker RJ, Brugman E, Roede MJ, Verloove-Vanhorick SP, Wit JM: Continuing positive secular growth change in The Netherlands 1955-1997. Pediatr Res 2000;47:316-323.

12 Knutsson U, Dahlgren J, Marcus C, Rosberg S, Bronnegard M, Stierna P, Albertsson-Wikland K: Circadian cortisol rhythms in healthy boys and girls: relationship with age, growth, body composition, and pubertal development. J Clin Endocrinol Metab 1997;82:536540.

13 Bailey D, Colantonio D, Kyriakopoulou L, Cohen AH, Chan MK, Armbruster D, Adeli $\mathrm{K}$ : Marked biological variance in endocrine and biochemical markers in childhood: establishment of pediatric reference intervals using healthy community children from the CALIPER cohort. Clin Chem 2013;59:1393-1405.
4 Elmlinger MW, Kuhnel W, Ranke MB: Reference ranges for serum concentrations of lutropin ( $\mathrm{LH})$, follitropin (FSH), estradiol $\left(\mathrm{E}_{2}\right)$, prolactin, progesterone, sex hormone-binding globulin (SHBG), dehydroepiandrosterone sulfate (DHEAS), cortisol and ferritin in neonates, children and young adults. Clin Chem Lab Med 2002;40:1151-1160.

15 Dettenborn L, Tietze A, Kirschbaum C, Stalder T: The assessment of cortisol in human hair: associations with sociodemographic variables and potential confounders. Stress 2012;15:578-588.

16 Karlén J, Frostell A, Theodorsson E, Faresjö $\mathrm{T}$, Ludvigsson J: Maternal influence on child HPA axis: a prospective study of cortisol levels in hair. Pediatrics 2013;132:e1333-e1340.
7 Barth JH: Normal hair growth in children. Pediatr Dermatol 1987;4:173-184.

18 Duggins OH, Trotter M: Age changes in head hair from birth to maturity. II. Medullation in hair of children. Am J Phys Anthropol 1950; 8:399-415.

19 Duggins OH, Trotter M: Changes in morphology of hair during childhood. Ann NY Acad Sci 1951;53:569-575.

20 Russell E, Koren G, Rieder M, Van Uum SH: The detection of cortisol in human sweat: implications for measurement of cortisol in hair. Ther Drug Monit 2014;36:30-34.

21 Albar WF, Russell EW, Koren G, Rieder MJ, Van Umm SH: Human hair cortisol analysis: comparison of the internationally-reported ELISA methods. Clin Invest Med 2013; 36:E312. 